\title{
OPTICAL-EXCITATION FUNCTION AND POLARIZATION OF 4227- $\AA$ RESONANCE LINE OF Ca EXCITED BY 8-500 eV ELECTRON IMPACT
}

\author{
D. Dziczek, D. Dyl, M. Piwiński and S. Chwirot \\ Institute of Physics, Nicholas Copernicus University \\ Grudziądzka 5-7, 87-100 Toruń, Poland
}

(Received January 21, 1998)

\begin{abstract}
We report on measurements of the relative optical-excitation function for the $4^{1} P$ state of $\mathrm{Ca}$ and polarization degree of the $4227-\AA$ resonance line. The measurements were carried out using crossed low-density beams of electrons and calcium atoms, for incident electron energies from 8 to $500 \mathrm{eV}$. For each electron energy we have used 28 results obtained at different polarizer positions from $0-180^{\circ}$ to extract the required parameters. Excellent agreement between the present results and earlier predictions has been found.
\end{abstract}

PACS numbers: $34.80 . D p$

\section{Introduction}

Excitation processes of metal atoms are of great interest for astrophysics and plasma physics $[1,2]$. In particular, the excitation functions of metal atoms are essential for analysis of stellar lines where in the absence of ion lines neutral resonance lines become very important. Calcium is for instance an element contributing many lines to spectra of solar-type stars and the Ca $4227-\AA$ line is one of the most important from the point of view of such applications.

Precise measurements of the polarization and relative optical-excitation function of the calcium $4227-\AA$ resonance line in the incident electron energy range of 3-1400 eV were reported by Ehlers and Gallagher [3]. Unfortunately, in the energy range of $8-100 \mathrm{eV}$ only 8 data points were measured and in the range of $100-500 \mathrm{eV}$ only 3 results are available. The aim of this paper is to complement those data using a similar method and to compare the obtained results with earlier predictions. We present 22 new measurements of the relative excitation function and polarization for electron excitation of the calcium $4227-\AA$ resonance line: 13 in the $8-100 \mathrm{eV}$ region and 9 in the range of $100-500 \mathrm{eV}$. 


\section{Experimental technique}

\subsection{Apparatus}

The experiment is housed in a cylindrical vacuum chamber made of stainless steel (dimensions: $67 \mathrm{~cm}$ in diameter by $56 \mathrm{~cm}$ in height). Typical operating pressure is of the order of $5 \times 10^{-7}$ mbar with the $\mathrm{Ca}$ atomic beam flowing into the chamber.

The beam of calcium atoms is produced by a stainless steel oven and collimated by a nozzle and a circular aperture in the magnetic shield covering the oven's body. Two bifilar sets of Thermocoax (Philips) wires are used to heat both the oven's main body and the nozzle. Such a geometry minimises the magnetic field produced by the heating currents, leaking into the interaction region. The main body of the oven is typically heated to $630^{\circ} \mathrm{C}$ and the nozzle is kept at a temperature of $720^{\circ} \mathrm{C}$. The temperatures remain constant within $1^{\circ} \mathrm{C}$ during the measurement. The atomic density at the interaction region located about $15 \mathrm{~mm}$ above the nozzle was estimated to be approximately $10^{11}$ atoms $/ \mathrm{cm}^{3}$. A liquid nitrogen trap placed over the oven prevents contamination of the chamber with $\mathrm{Ca}$ atoms. The oven is separated from the rest of the system by a water-cooled heat-sink to absorb its thermal radiation, and a $\mu$-metal shield is used to prevent a field produced by oven heaters from entering the chamber.

The electron beam is produced by a commercial electron gun (EG-402EL, Comstock) providing stable currents of about $2 \mu \mathrm{A}$ for the electron energy of $500 \mathrm{eV}$ and $0.1 \mu \mathrm{A}$ for $8 \mathrm{eV}$. The electrons after passing through the uniform density region of the calcium beam are collected with Faraday cup and the actual electron current is measured using nano-ammeter. Care was taken to reduce possible sources of systematic errors with all the typical precautions like shielding the possible sources of stray electric fields and coating all the surfaces in the vicinity of the interaction region with colloidal graphite. The incident electron energy is determined by a bias voltage applied to the cathode of the electron gun. The electron energy spread of the electron beam used in this experiment was below $0.4 \mathrm{eV}$ FWHM, as determined by the previous measurement using an electron energy analyser. The maximum acceptance angle for the electron gun $(\Theta)$, as defined by the beam apertures, is $0.19 \mathrm{rad}$.

Photons emitted in a direction perpendicular to the direction defined by the momentum of the incident electron beam and the calcium beam are collected by a quartz lens positioned in such a way that the interaction region is at its focus. The size of the interaction region as determined by the geometry of the electron and calcium beams, changes slightly with the incident electron energy and its maximum volume is $3 \mathrm{~mm}^{3}$. The maximum acceptance angle for the photons $(\Psi)$, as determined by the size of the lens, is $0.15 \mathrm{rad}$. The fluorescence light collected by the lens passes through a polarization analyser and appropriate interference filter and then is detected by a photomultiplier (9235QSB, THORN EMI). Pulses from the photomultiplier are amplified (EG\&G Ortec preamplifier 9301), fed into constant fraction discriminator (EG\&G Ortec 935) and counted by a suitable scaler. 
The polarization analyser is composed of a $\lambda / 4$ retarder (mica, Spindler $\&$ Hoyer, retardation tolerance $5 \mathrm{~nm}$ ) and a linear polarizer (Spindler \& Hoyer). A measured ratio of the parallel to perpendicular transmittances for this polarizer at wavelength of $4227-\AA$, is $K_{\|} / K_{\perp}=117 \pm 10.0$. The linear polarizer is followed by another $\lambda / 4$ plate to ensure that the light emerging from the polarising optics is always circularly polarised and the measured photon fluxes are not influenced by polarization sensitivity of the photomultiplier. The linear polarizer and the second retarder are rotated together in the range of $0-180^{\circ}$ in 28 steps. The positioning is controlled by a stepper motor referenced to a zero position using a slotted optoswitch. The accuracy of the positioning of the linear polarizer is approximately $0.6^{\circ}$.

\subsection{Procedure}

At a given incident electron energy and at each polarizer position photon pulses from the photomultiplier are counted by a digital counter and stored for further processing. Additionally, the actual electron current passing through the calcium beam, photon pulses due to the background (including dark counts of photomultiplier) and the data collected at the incident electron energy of $100 \mathrm{eV}$ are also stored. Typical signals were on the order of $10^{2}-10^{3} \mathrm{cps}$ while the background counting rate was $40-50 \mathrm{cps}$ including $20-25 \mathrm{cps}$ of thermal noise pulses. After background subtraction all the results have been normalised to the initial calcium beam density and to an electron current of $100 \mathrm{nA}$. For each incident electron energy, contrary to the procedure described in Ref. [3], we use all the 28 results (at each polarizer position) and an appropriate nonlinear least-squares fitting procedure to extract the total number of photomultiplier counts obtained with the polarizer axis parallel $\left(I_{\|}\right)$and perpendicular $\left(I_{\perp}\right)$ to the electron beam. This is enough to calculate the measured polarization degree $P_{m}=\left(I_{\|}-I_{\perp}\right) /\left(I_{\|}+I_{\perp}\right)$ and the measured relative apparent excitation function $R(E)$ (the terminology is that proposed in Ref. [4]). Several corrections, due primarily to the imperfection of the polarization analyser, the finite acceptance angles for the photons and electrons, and also to the anisotropy of emitted radiation must be applied to these raw data to obtain the polarization and excitation functions $[3,4]$. If $\bar{I}$ is the average intensity per unit solid angle and $I_{90}$ is the observed light intensity, then in the small-angle approximation

$$
I_{90}=\bar{I}\{3[1-(\varepsilon+\delta) P] /(3-P)\},
$$

where $\varepsilon=\Psi^{2} / 4, \delta=\Theta^{2} / 4$ and the corrected polarization $P$ is given in terms of measured polarization as

$$
P_{m}=\left(\frac{K_{\|}-K_{\perp}}{K_{\|}+K_{\perp}}\right)\left(\frac{1-\varepsilon}{1-\varepsilon P}\right)\left(\frac{1-3 \delta}{1-\delta P}\right) P
$$

\section{Results and discussion}

Our final results for the corrected polarization and the relative optical excitation function (relative to the value measured at $98.7 \mathrm{eV}$ ) are shown in Figs. 1 and 2 . The radii of the data points represent approximately the uncertainties due 
to the counting statistics, the fitting procedure and to the errors of the correction factors. The total error for the polarization degree is estimated to be less than $1.5 \%$ while for the $R(E)$ it is less than 0.01 . Data taken repeatedly for the same electron energies on different days, after cleaning and reassembling the apparatus, generally agreed within the uncertainties given above.

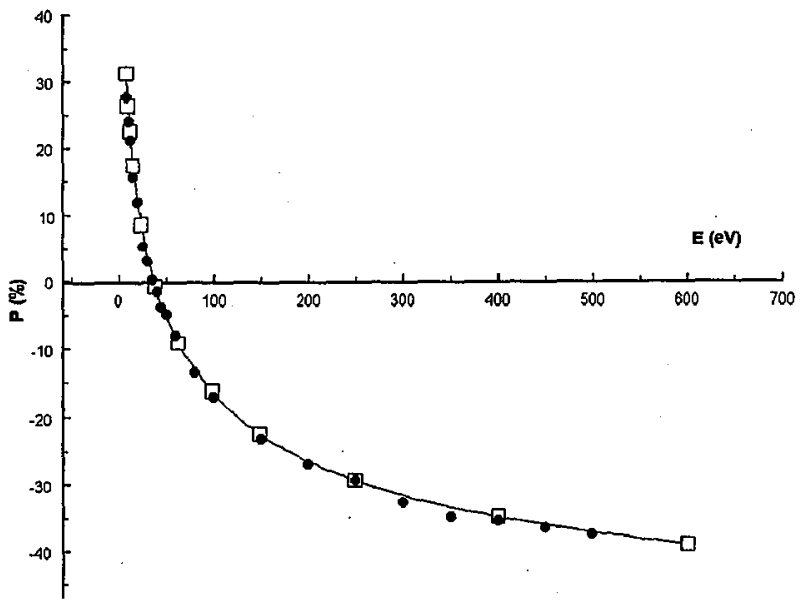

Fig. 1. The polarization for calcium $4227-\AA$ radiation ( $\bullet-$ present work, $\square-R e f$. [3]).

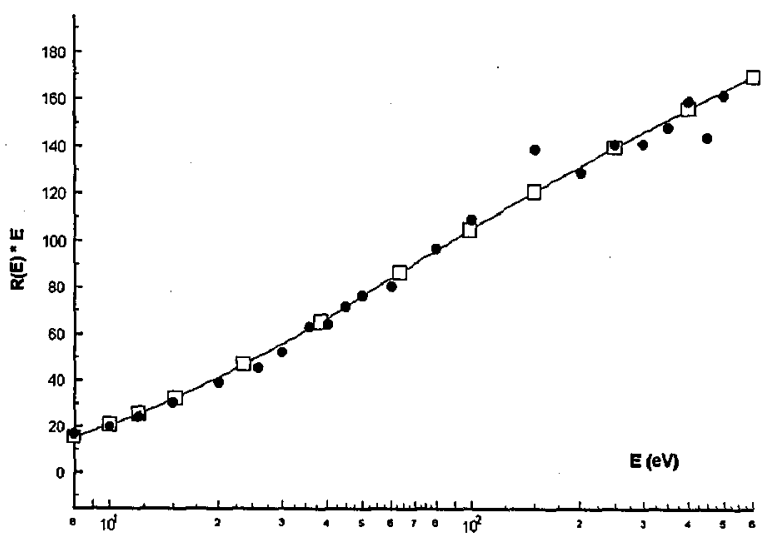

Fig. 2. The corrected relative optical-excitation function multiplied by electron energy in $\left(a_{0}^{2} \pi \mathrm{eV}\right)(\bullet-$ present work, $\square-\operatorname{Ref} .[3])$.

We compare our results with the data reported in Ref. [3] for the incident electron energy $8-500 \mathrm{eV}$. The agreement between the present results and interpolation based on the earlier measurements (solid lines) is almost perfect, especially for the polarization degree. In the case of the relative optical excitation function 
agreement is also very good, although $R(E)$ is much more sensitive to possible small fluctuations of the calcium beam density than the degree of polarization.

The present work is only the second experimental study of the relative optical-excitation function and the polarization of the $\mathrm{Ca} 4227-\AA$ resonance line. Our results fully confirm the earlier data of Ref. [3]. Measurements of the polarization degree for 22 incident electron energies in the range of 8-500 eV complement earlier results, especially for incident electron energies below $100 \mathrm{eV}$. We believe they can be of some value for those interested in electron excitation of calcium atoms in this incident electron energy range.

\section{Acknowledgments}

This work was supported by the Committee for Scientific Research (grants No. 2 P03B 06810 and No. 2 P03B 068 09).

\section{References}

[1] G. Smith, J. Phys. B, Al. Mol. Opt. Phys. 21, 2872 (1988).

[2] L.H. Aller, Astrophysics, 2nd edition, Ronald, New York 1963.

[3] V.J. Ehlers, A. Gallagher, Phys. Rev. A 7, 1573 (1973).

[4] B.L. Moiseiwitsch, S.J. Smith, Rev. Mod. Phys. 40, 238 (1968). 